\title{
Role of Cardiac Magnetic Resonance Imaging in Diagnosis of Myocardial Infarction
}

\author{
Ahmed Mohamed Abdel Hakam*, Hisham Mostafa Imam, Hany Mohamed Abdel-Hakeem Seif, \\ Nisreen Adel Abbas
}

Department of Diagnostic Radiology, Faculty of Medicine, Assiut University, Assiut, Egypt

Email address:

ahmed7akam@yahoo.com (A. M. A. Hakam)

*Corresponding author

\section{To cite this article:}

Ahmed Mohamed Abdel Hakam, Hisham Mostafa Imam, Hany Mohamed Abdel-Hakeem Seif, Nisreen Adel Abbas. Role of Cardiac Magnetic Resonance Imaging in Diagnosis of Myocardial Infarction. International Journal of Medical Imaging.

Vol. 8, No. 4, 2020, pp. 126-137. doi: 10.11648/j.ijmi.20200804.19

Received: January 27, 2020; Accepted: February 20, 2020; Published: December 28, 2020

\begin{abstract}
Introduction: Cardiac Magnetic Resonance Imaging provides an accurate and reproducible modality for the assessment of global ventricular volumes and function, and valuable in establishing a diagnosis of chronic and acute MI and distinguishing this condition from non-ischemic cardiomyopathies. Aim: To evaluate the role of cardiac Magnetic Resonance Imaging technique in diagnosis of myocardial infarction, assessment of myocardial viability. Subject and Method: The study was conducted over a period of 3 years at Assiut University Hospital. Fifty-six patients were recruited. All patients were subjected to full history taking and radiological evaluation using a 1.5-T MR system and echocardiography. Agreement regarding segmental wall motion was assessed by using Cohen's Kappa statistics. Also ejection fraction measurement by both modalities was compared using bland-Altman plots. Agreement between Magnetic Resonance Imaging and conventional coronary angiography in detection of diseased coronary arteries was examined using Cohen's kappa coefficient $(\kappa)$. Results: It was found that ejection fraction detected by Echo were overestimated relative to Magnetic Resonance Imaging with statistically significant difference $(\mathrm{p}=0.004)$. While LV diameters detected by echocardiography were underestimated relative to Magnetic Resonance Imaging. Good agreement between Magnetic Resonance Imaging and Echo regarding segmental wall motion. There was positive very high correlation between the two modalities in evaluation of LV Functionality indices. Conclusion: CMR is superior to echo in evaluating left ventricular functionality indices with echo underestimating these indices except the ejection fraction which is overestimated by Echo. Moreover, CMR is better in evaluating post MI complications and associated cardiac abnormalities.
\end{abstract}

Keywords: Myocardial Infarction (MI), Cardiac Magnetic Resonance (CMR) and Echocardiography (ECHO), Left Ventricular (LV) and Late Gadolinium Enhancement (LGE)

\section{Introduction}

Although myocardial infarction (MI) is one of the major causes of morbidity Worldwide, mortality has decreased significantly and this drop appears to be the result of the decline in the incidence of ST-segment elevation myocardial infarction (STEMI) along with the absolute reduction of overall mortality of MI [1]. The reduction of mortality for MI is due to the efficacy of current therapeutic strategies focused on an early reopening of the infarct-related artery, either by thrombolytic therapy or primary percutaneous coronary intervention (PCI) [2].
The current diagnostic tools, blood biomarkers (e.g. troponin), electrocardiography, and ultrasonography - are the key tools in the diagnosis of acute myocardial infarction (AMI) or what is recently called acute coronary syndrome (ACS) but these markers provide only a partial insight in the complex, evolving processes occurring in the jeopardized myocardium [3]. The accurate diagnosis and differentiation of ACS from other acute cardiac diseases which is essential for therapeutic decision making and specific therapies may be challenging or even impossible with the above diagnostic tools [3].

Among different imaging techniques, magnetic resonance (MR) imaging has an important role in work-up of acute chest pain with uncertain diagnosis, for risk stratification, and to 
identify patients at high risk of further events such as reinfarction or heart failure [4]. Moreover, cardiac MRI provides an accurate and reproducible modality for the assessment of global ventricular volumes and function, and valuable in establishing a diagnosis of chronic MI and distinguishing this condition from non-ischemic cardiomyopathies [5].

The aim of this study was to evaluate the role of cardiac MR imaging (CMR) technique in diagnosis of MI and assessment of myocardial viability as well as for risk stratification to evaluate prognostic outcome and to shed the light on common complications of MI.

\section{Materials and Methods}

\subsection{Study Population}

Over a period of three years (from February 2016 till June 2019), CMR study was performed on 56 patients divided into two groups:

a. Group A: included 28 patients known to be ischemic cardiomyopathy based on clinical symptoms and documented LV dysfunction by echocardiography. They had history of coronary artery bypass graft "CABG" (7 patients), percutaneous coronary intervention "PCI" (10 patients) or history of myocardial infarction (11 patients). These patients who had experienced prior myocardial infarction were examined by coronary angiography with subsequent CMR within a mean time interval of 16 days.

b. Group B: included 28 patients with first presentation of acute chest pain and raised cardiac enzymes with no previous history of myocardial infarction.

Patients with hemodynamic instability, atrial fibrillation, partial heart block, general contraindications to MRI e.g., implanted devices and contraindication for contrast material including known allergy and renal insufficiency were excluded from the study.

All patients were subjected to full 2D echocardiography examination with estimation of $\mathrm{EF}$ and segmental wall motion abnormalities at cardiology unit of internal medicine department. Also, evaluation of any associated chamber enlargement or valvular lesions. CMR imaging was carried out in Radio-diagnosis department, Assiut University Hospital. Patients were recruited from the Cardiology Unit of Internal Medicine Department in Assiut University Hospital.

\subsection{Echocardiogram Imaging Protocol}

Echo imaging (Philips sonos 5500, Andover, MA) was performed with a transducer of 2.5-4.0 MHz. Images were obtained in short axis, 4- and 2-chamber views.

\subsection{MR Imaging Protocol}

The study was performed at two non-identical 1.5-T MR system in MRI Unit of the Radiology Department of Assiut university hospitals as 44 patients underwent the MRI examination by Philips 1.5 Tesla system (Achieva, Medical Systems, The Netherlands) while the remaining 12 patients underwent the MRI examination by Siemens 1.5 Tesla.
Localizer sequences in three orthogonal planes were taken followed by standard cardiac 2-, 3-, and 4-chamber views as well as short axis (SA) cine images in steady state free precession (SSFP). All images were acquired using a phasedarray cardiac coils (8 elements phased-array coil, receive only, Philips) \& (6 elements phased-array coil, receive only, Siemens) during single breath-holds (end expiratory of about 9-13 s) with ECG gating. Acquisition parameters for functional Cine images were; repetition time msec/echo time msec 2.9/1.4, flip angle $60^{\circ}$, slice thickness $8 \mathrm{~mm}$, slice number $9-11$, Field of view (FOV) $320 \mathrm{~mm}^{2}$ and matrix $160 \times 256$. Contrast media was injected of about $0.2 \mathrm{mmol} / \mathrm{kg}$ body weight with acquisition of real-time retrospective gated dynamic cine SA view in five slices in free breathing fashion (at rest). Then, late gadolinium enhancement (LGE) images were obtained $10 \mathrm{~min}$ after the injection of the contrast material using an inversion recovery (IR) sequence (phase sensitive inversion recovery is the most reliable sequence).

\section{Data Processing for MRI}

\subsection{Functional Image Analysis}

Short axis CINE images were transferred to a workstation equipped with a dedicated cardiac software for further postprocessing analysis, two different post-processing softwares were used namely, Extended workspace "EWS"; Nederland B. V. Best, Netherlands (Philips Medical Systems) and Synapse 3D Fujifilm's software. Delineation of endo- and epicardial contours of left ventricle at end-diastole and endsystole gives global functional parameters (LVEDV, LVESV, LVEDD, LVESD and EF). Regional function was also assessed qualitatively (described as normal, hypokinetic, akinetic, or dyskinetic) using the 17-segment model of the American Heart Association (AHA).

\subsection{Tissue Characterization \& Viability Analysis}

Visual assessment of presence scar tissue and its extent across myocardial wall was performed For Risk stratification, As Transmural extent of infarction can be expressed as follows: Grade $0=$ no hyperenhancement, Grade $1 \& 2=$ less than 50\% LGE of left-ventricular wall thickness and Grade 3 \& $4=$ more than $50 \%$ LGE of left-ventricular wall thickness.

\subsection{Estimation of Infarct Size}

Manual Delineation of the infarct territory involves identifying the border of the bright infarct zone, distinguished from the lower signal intensity of neighboring unaffected tissue and the remote zone. The manual delineation based on visual assessment. This approach is subjective, but it minimizes the potential for auto-mated error.

\subsection{Statistical Methods}

Date entry and data analysis were conducted using SPSS21 (IBM Corp. Released 2012. IBM SPSS Statistics for Windows. Armonk, NY, USA). The Shapiro-wilk test was 
used to test the normality of numerical data distribution. Continuous data were expressed as mean \pm standard deviation (SD) or median with ranges and compared using student's t-test. Chi-square or Fisher exact tests were used to compare between qualitative variables. Agreement between MRI and ECHO in segmental wall motion was examined using Cohen's kappa coefficient ( $\kappa)$. Segmental wall motion in MRI and ECHO was assessed by a 4-point scale (1, normal; 2, hypokinesia; 3, akinesia; 4, dyskinesia) in a per segment analysis (with the 17-segment model). To examine agreement between EF in MRI and ECHO, Bland-Altman plots were used. To determine the diagnostic value of MRI, a series of $2 \times 2$ contingency tables were constructed to contrast the examination tool of interest (CMR) to standard reference tool (ECHO) as regards left atrial (LA) enlargement, left ventricular (LV) enlargement, wall thinning, intracavitary thrombus and valvular lesions. Then Negative predictive values (NPV) as well as 95\% CI were obtained. Agreement between MRI and conventional coronary angiography in detection of diseased coronary arteries was examined using Cohen's kappa coefficient ( $(\kappa)$. P-value considered statistically significant when $\mathrm{P} \leq 0.05$.

\section{Ethical Considerations}

The study protocol was approved by the Institutional Research Ethics committee, faculty of medicine, Assiut University and after taking written informed consent from all patients, these patients were subjected to full clinical history. No expected risk on patients as dealing with patient will be limited to collection of serum samples and confidentiality was assured. Statement describing the research procedure to be given to the participants will be taken from participants before being included in the study. Informed consent will be taken from participants before being included in the study.

\section{Results}

Table 1 revealed the baseline characteristics of both study groups. There were 42 (75\%) males and 14 (25\%) females with mean age of group A was $59.1 \pm 7.5$ while the mean age of group B was $53.5 \pm 11.8$, ranging from 40 to 70 years. All the patients in this study had one or more risk factors in both groups such as diabetes mellitus (78.6\% and 57.1\%), arterial hypertension $(57.1 \%$ and $57.1 \%)$, smoking $(60.7 \%$ and $57.1 \%)$ and obesity with BMI $\geq 25(100 \%$ and $75 \%)$.

Table 1. Baseline Clinical Characteristics of Study groups.

\begin{tabular}{lll}
\hline Group A (CMI) $(\mathrm{n}=28)$ & & \\
Age in years & Mean \pm SD & $59.07 \pm 7.5$ \\
& Median (IQR) & $58(7)$ \\
Sex & Male & $21(75 \%)$ \\
& Female & $7(25 \%)$ \\
BMI & Mean \pm SD & $28.89 \pm 2.4$ \\
Risk factors & Median (Range) & $28.5(4)$ \\
Hypertension & & \\
Diabetes Mellitus & & $16(57.1 \%)$ \\
Smoking & & $22(78.6 \%)$ \\
Overweight/Obesity $(\mathrm{BMI} \geq 25)$ & & $17(60.7 \%)$ \\
Group B (AMI) $(\mathrm{n}=28)$ & & $28(100 \%)$ \\
\hline
\end{tabular}

\begin{tabular}{lll}
\hline Age in years & Mean \pm SD & $53.50 \pm 11.8$ \\
& Median (IQR) & $56.5(19)$ \\
Sex & Male & $21(75 \%)$ \\
& Female & $7(25 \%)$ \\
BMI & Mean \pm SD & $26.46 \pm 3.1$ \\
Risk factors & Median (Range) & $26.5(4)$ \\
Hypertension & & \\
Diabetes Mellitus & & $16(57.1 \%)$ \\
Smoking & & $16(57.1 \%)$ \\
Overweight/Obesity $(\mathrm{BMI} \geq 25)$ & & $16(57.1 \%)$ \\
\hline
\end{tabular}

According to the echo data in both groups, the mean LVESD was $4.3 \mathrm{~cm}$ and $3.6 \mathrm{~cm}$ while the mean LVEDD was $5.4 \mathrm{~cm}$ and $4.7 \mathrm{~cm}$, respectively. The mean EF was $32.3 \%$ and $40.1 \%$. WM abnormality was $100 \%$ and $75 \%$. The sum of WM abnormality segments was calculated in both groups (A and B). Hypokinetic segments were presented in $36.8 \%$ and $65.1 \%$ while the akinetic segments were presented in $53.8 \%$ and $18.1 \%$. Regarding the CMR data in both groups (A and B), the mean LVESD was $4.4 \mathrm{~cm}$ and $3.7 \mathrm{~cm}$ while the mean LVEDD was $5.6 \mathrm{~cm}$ and $4.8 \mathrm{~cm}$. Also, the mean EF was $30.4 \%$ and $39.1 \%$. The mean LVESV was $170.6 \mathrm{~mL}$ and $97.5 \mathrm{~mL}$ while the mean LVEDV was $237.1 \mathrm{~mL}$ and $154.3 \mathrm{~mL}$. WM abnormality was $(96.4 \%$ and $100 \%)$. The sum of WM abnormality segments as revealed in figures 1 and 2 was as follows: hypokinetic segments were presented in $40 \%$ and $65.9 \%$ while the akinetic segments were presented in $47.3 \%$ and $25.3 \%$, respectively.

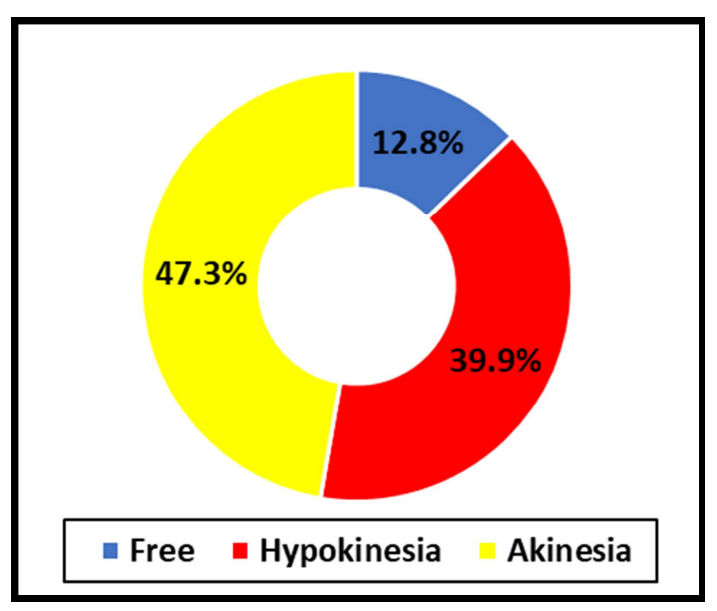

Figure 1. WM abnormality segments among the studied CMI Cases.

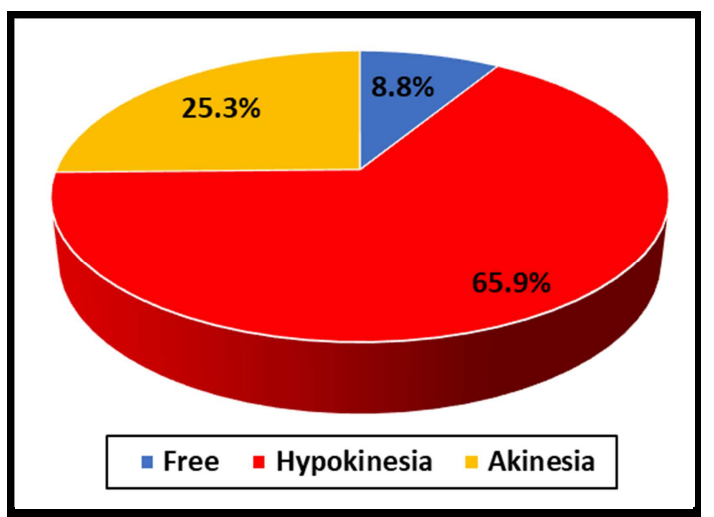

Figure 2. WM abnormality segments among the studied AMI Cases. 
Myocardial segment viability of the patients in group A (table 2) that estimated via LGE technique revealed that patients with LGE $<50 \%$ who were eligible for revascularization procedures represented about $39.3 \%$. While patients with LGE $>50 \%$ who were not eligible for revascularization procedures and not having any viable segment represented $60.7 \%$. It was found that (LAD) was the most diseased in this group $78.6 \%$ followed by (RCA) was 50\%, and (LCX) was 39.3\%. The mean infarct size core was 22.96 in this group and 16 patients have post MI complications $(57.1 \%)$.

Table 2. Cardiac MRI data of Study groups.

\begin{tabular}{lll}
\hline Group A (CMI) $(\mathbf{n}=\mathbf{2 8})$ & & $11(39.3 \%)$ \\
\hline Myocardial Segment Viability & LGE $<50 \%$ (VIABLE) & $17(60.7 \%)$ \\
& LGE $>50 \%$ (Non-VIABLE) & $22(78.6 \%)$ \\
Coronary territories Affected & LAD & $11(39.3 \%)$ \\
& LCX & $14(50 \%)$ \\
Post MI Complication & RCA & $16(57.1 \%)$ \\
Group B (AMI) $(\mathrm{n}=28)$ & & $5(17.8 \%)$ \\
& & $8(28.6 \%)$ \\
Pattern of Enhancement & Normal & $15(53.6 \%)$ \\
& Non-ischemic & $25(89.3 \%)$ \\
Edema at T2 & Ischemic & $22.96 \pm 14.6$ \\
Infarction Size $\%$ & Yes & $5(33.3 \%)$ \\
Salvaged Index Ratio (SIR) of ischemic patients $(\mathrm{n}=15)$ & Mean \pm SD & $10(66.7 \%)$ \\
& Poor & $9(60 \%)$ \\
Myocardial Segment Viability of ischemic patients (n=15) & Good & $6(40 \%)$ \\
& $<50 \%$ & $8(53.3 \%)$ \\
Coronary Territories Affected of ischemic patients (n=15) & $>50 \%$ & $4(26.6 \%)$ \\
& LAD & $5(33.3 \%)$ \\
Microvascular obstruction (MVO) & LCX & $8(28.6 \%)$ \\
Post MI Complication & RCA & $9(32.1 \%)$ \\
\hline
\end{tabular}

Regarding the pattern of LGE in group B (table 2 and figures 3 and 4), it was revealed that patients with ischemic CMR criteria represented about 54\%. According to the myocardial segment viability of the patients in this group that estimated via LGE technique revealed that the patients with LGE $<50$ represented about $60 \%$. While, patients with LGE $>50 \%$ represented $40 \%$. It was found that (LAD) was the most diseased in this group 53.3\% followed by RCA was $33.3 \%$, and (LCX) was $26.6 \%$. The mean infarct size core was 22.96 in this group and the patients who have post MI complications were $32.1 \%$ and those who have MVO were $28.6 \%$. The estimated SIR of the ischemic patients revealed that patients who had good SIR were $66.7 \%$.

It was found that the EFs detected by echocardiography were overestimated relative to MRI with statistically significant difference $(p=0.004)$. LV diameters detected by echocardiography were underestimated relative to MRI with statistically significant difference with $(p=0.003)$. CMR was much more sensitive than Echo to detection of segmental wall motion abnormalities $(\mathrm{p}<0.001)$.

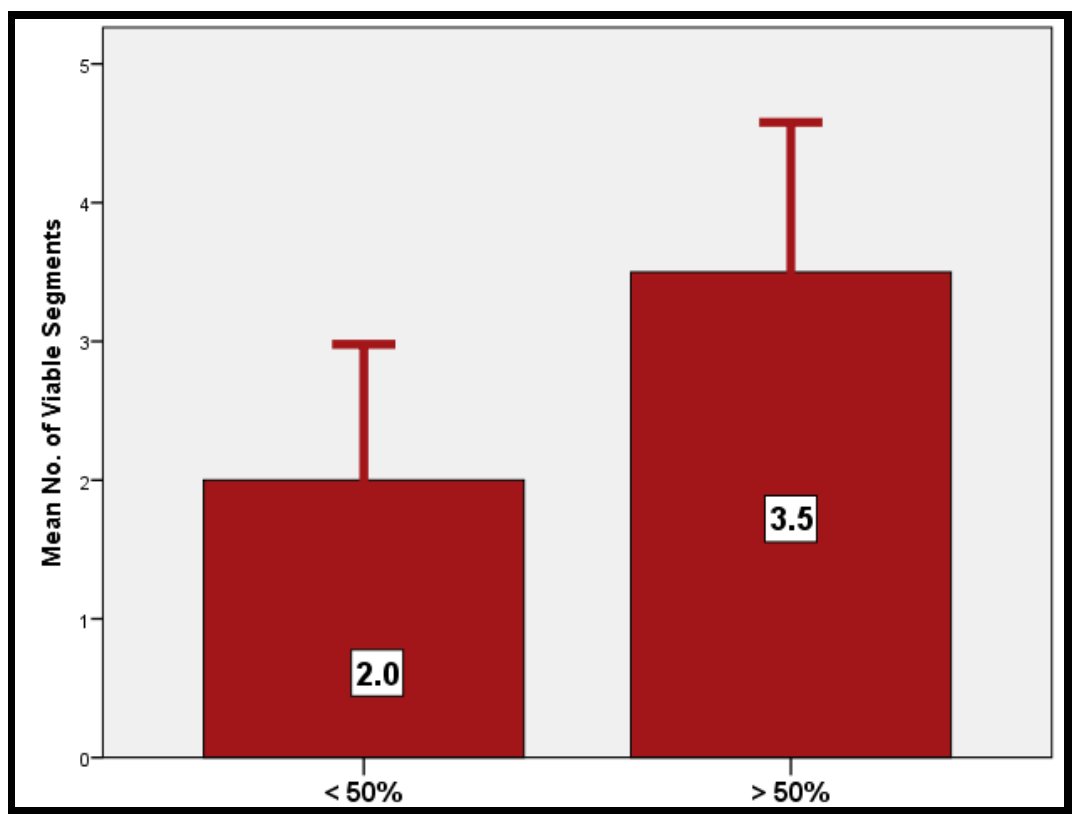

Figure 3. Mean Viability Segment No. by MRI among the studied CMI Cases. 


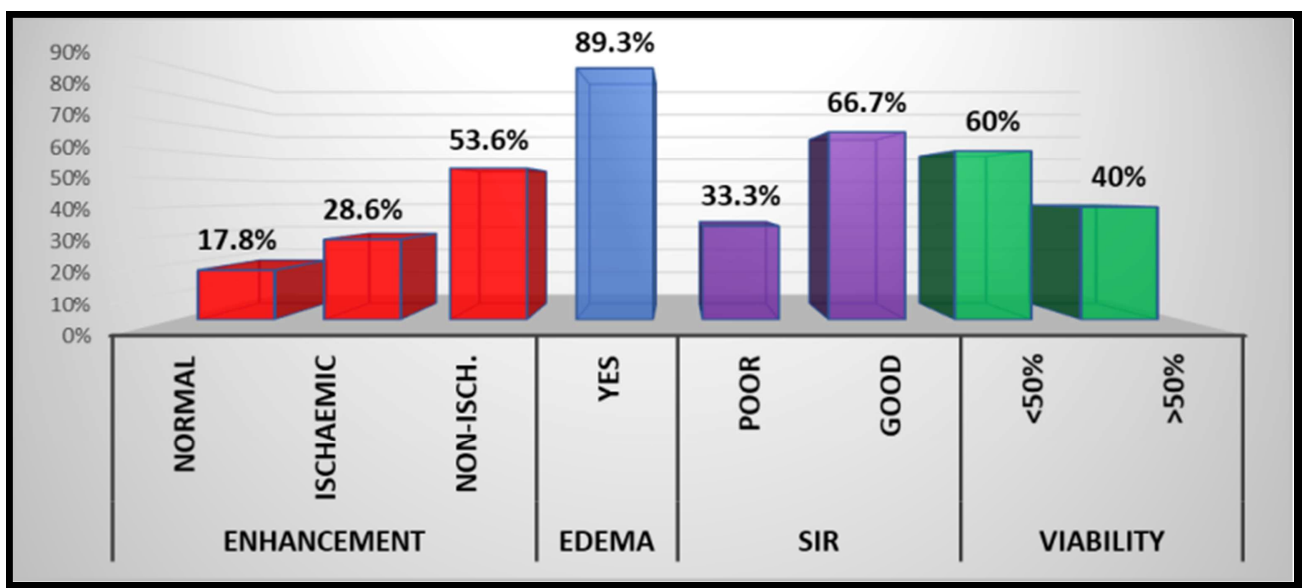

Figure 4. Cardiac MRI Parameters of Study CMI Cases.

Agreement between Echocardiography and MRI for the estimation of ejection fraction (EF) was noted. Among the 56 patients, the mean EF by MRI was $34.8 \%$ and the mean EF by echocardiography was $36.2 \%$ with a mean difference of $4.6 \%$. SD of bias was of 3.67 and $95 \%$ limits of agreement were 9.94: -7.83 (Table 3 and Figure 5).

Table 3. Agreement between echocardiography and CMRI Regarding Ejection Fraction Percentage Estimation: A Bland-Altman plot $(n=56)$.

\begin{tabular}{ll}
\hline Measure & Value \\
\hline EF by echocardiography $(\%) *$ & $36.2(11.5)$ \\
EF by CMRI $(\%)$ & $34.8(14.5)$ \\
Bias** & 4.63 \\
SD of bias & 3.67 \\
$95 \%$ Limits of Agreement*** & $9.94:-7.83$ \\
\hline
\end{tabular}

*EF is expressed as mean (SD).

$* *$ Bias $=$ average of differences

$* * * 95 \%$ Limits of Agreement $=$ bias plus or minus 1.96 times SD

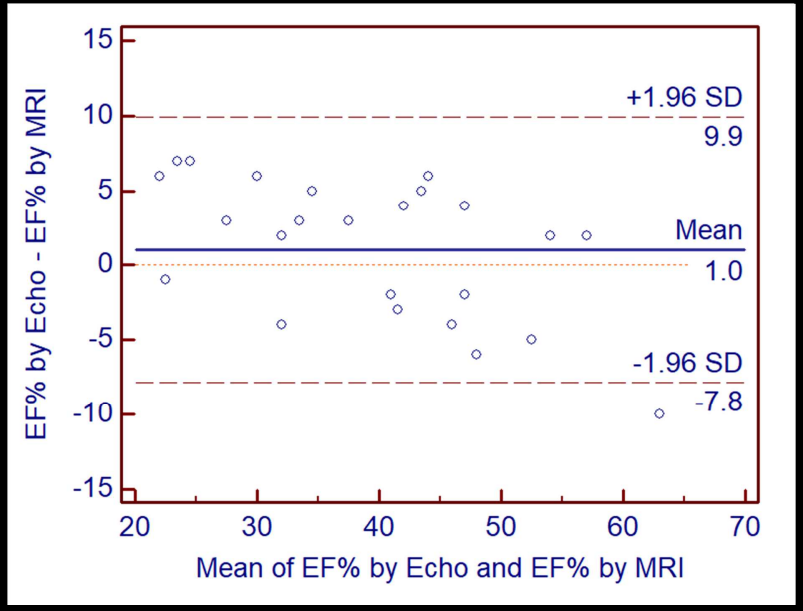

Figure 5. Bland Altman Agreement between Echo and CMRI for EF in CMI Cases.

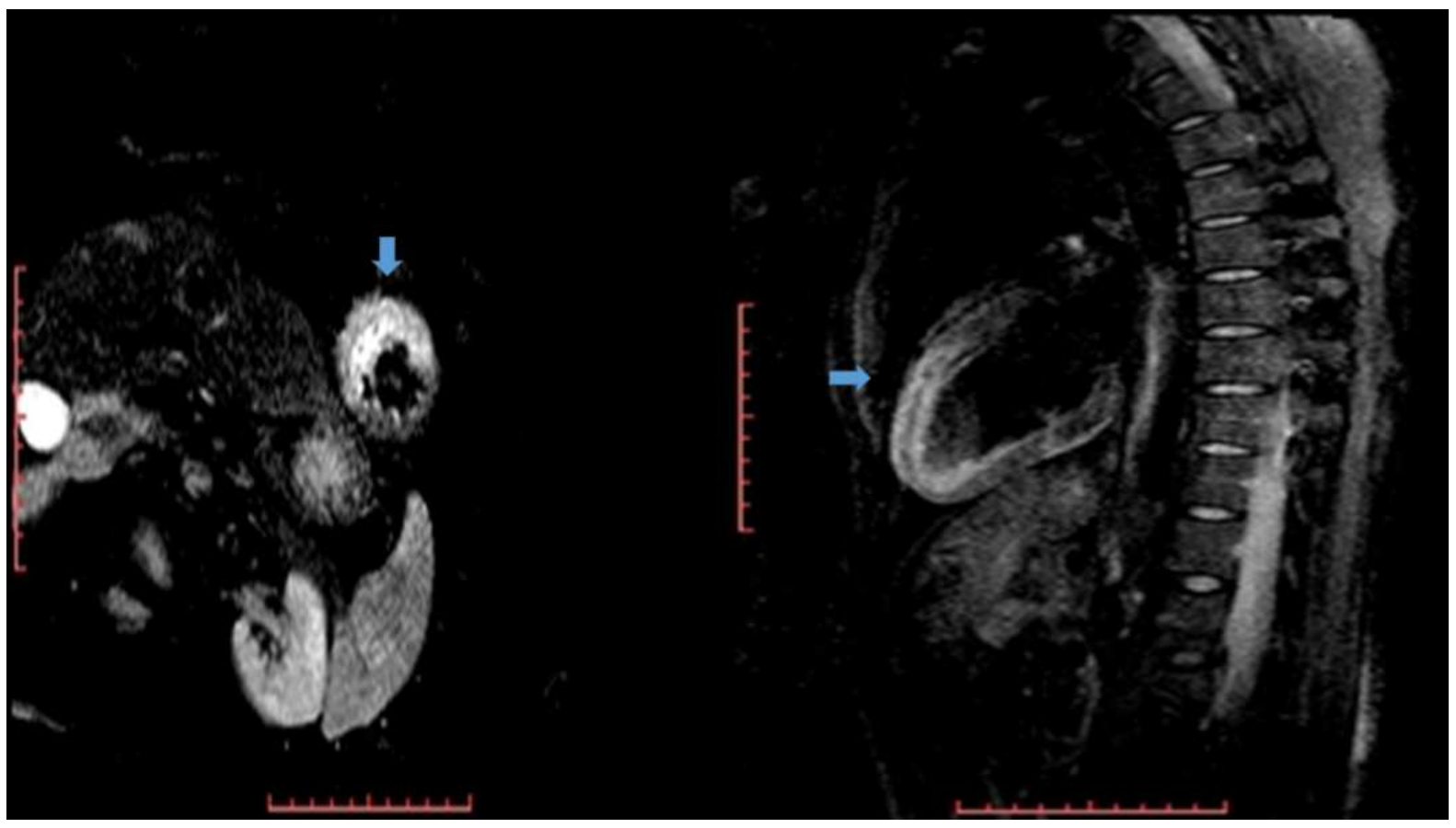

Figure 6. CMR findings. T2 fat saturation technique in short axis \& two Heart chamber planes. 
Table 4. Agreement between MRI and Conventional Coronary Angiography in detection of diseased coronary arteries in patients with chronic myocardial infarction (CMI).

\begin{tabular}{|c|c|c|c|c|c|c|c|c|}
\hline & \multicolumn{7}{|c|}{ Cardia MRI } & \multirow{2}{*}{ Total } \\
\hline & & 1 & 2 & 3 & 4 & 5 & 6 & \\
\hline \multirow{6}{*}{$\begin{array}{l}\text { Conventional } \\
\text { Coronary } \\
\text { Angiography }\end{array}$} & 1 & $7(25.9 \%)$ & $0(0 \%)$ & $0(0 \%)$ & $0(0 \%)$ & $0(0 \%)$ & $0(0 \%)$ & $7(25.9 \%)$ \\
\hline & 2 & $1(3.7 \%)$ & $3(11.1 \%)$ & $0(0 \%)$ & $0(0 \%)$ & $0(0 \%)$ & $0(0 \%)$ & $4(14.8 \%)$ \\
\hline & 3 & $2(7.4 \%)$ & $0(0 \%)$ & $5(18.5 \%)$ & $0(0 \%)$ & $0(0 \%)$ & $0(0 \%)$ & $7(25.9 \%)$ \\
\hline & 4 & $0(0 \%)$ & $0(0 \%)$ & $1(3.7 \%)$ & $3(11.1 \%)$ & $0(0 \%)$ & $0(0 \%)$ & $4(14.8 \%)$ \\
\hline & 5 & $0(0 \%)$ & $0(0 \%)$ & $0(0 \%)$ & $0(0 \%)$ & $2(7.4 \%)$ & $0(0 \%)$ & $2(7.4 \%)$ \\
\hline & 6 & $0(0 \%)$ & $0(0 \%)$ & $0(0 \%)$ & $0(0 \%)$ & $0(0 \%)$ & $3(11.1 \%)$ & $3(11.1 \%)$ \\
\hline \multirow{2}{*}{\multicolumn{2}{|c|}{$\begin{array}{l}\text { Total } \\
\text { Kappa Agreement }\end{array}$}} & $10(37 \%)$ & $3(11.1 \%)$ & $6(22.2 \%)$ & $3(11.1 \%)$ & $2(7.4 \%)$ & $3(11.1 \%)$ & $28(100 \%)$ \\
\hline & & & & 0.814 & & $\mathrm{P}<0.001$ & & \\
\hline \multicolumn{2}{|l|}{ Chi-square test } & & & 105.810 & & $\mathrm{P}<0.001$ & & \\
\hline
\end{tabular}

$1=\mathrm{LAD}, 2=\mathrm{LAD}+\mathrm{LCX}, 3=\mathrm{LAD}+\mathrm{RCA}, 4=\mathrm{LAD}+\mathrm{LCX}+\mathrm{RCA}, 5=\mathrm{LCX}$ and $6=\mathrm{RCA}$

Table 4 showed there was positive very high correlation between the two modalities (CMR and 2D Echo) in evaluation of LV Functionality indices (WMA Segment Number, LVESD, LVEDD and LVEF) with Pearson's Correlation Coefficient $(0.824,0.914,0.969$ and 0.952 respectively) and statistically significant difference in all parameters $(\mathrm{p}<0.001)$.

Moreover, there was good agreement between CMR and echo regarding segmental wall motion with Cohen's Kappa was $0.76, P=0.011$. Per-segment agreement between echocardiography and MRI as regards the detection of segmental WM abnormality in the 476 segments in our study, it was found that there was high agreement between the two modalities in evaluation of the SWMA as both cardiac MRI and $2 \mathrm{D}$ Echo detected $1.8 \%$ with no motion abnormality, hypokinesia in $28.6 \%$ of patients and Akinesia in $3.6 \%$ of total patients (Figure 6).

This current study reported that there was an agreement between the two modalities (CMR and conventional coronary angiography) with very high kappa agreement (0.814, $\mathrm{p}=0.001$ as shown in (table 5). Both modalities detected (LAD) affected territory only in $26 \%$ of the 28 patients while (LAD \& LCX) diseased territories seen in $11 \%$ and (LAD \& RCA) diseased territories seen in $18.5 \%$. It also revealed that true positive was about $82.1 \%$ and true negative was $7.1 \%$ while false positive and false negative were $7.1 \%$ and $3.7 \%$ respectively (Figure 7).

Table 5. Diagnostic accuracy of MRI in detection of diseased coronary arteries in patients with chronic myocardial infarction (CMI) using Conventional Coronary Angiography as a reference standard.

\begin{tabular}{lll}
\hline & $\mathbf{n = 2 8}$ & $\mathbf{\%}$ \\
\hline True Positive & 23 & $82.1 \%$ \\
True Negative & 2 & $7.1 \%$ \\
False Positive & 2 & $7.1 \%$ \\
False Negative & 1 & $3.7 \%$ \\
\hline
\end{tabular}

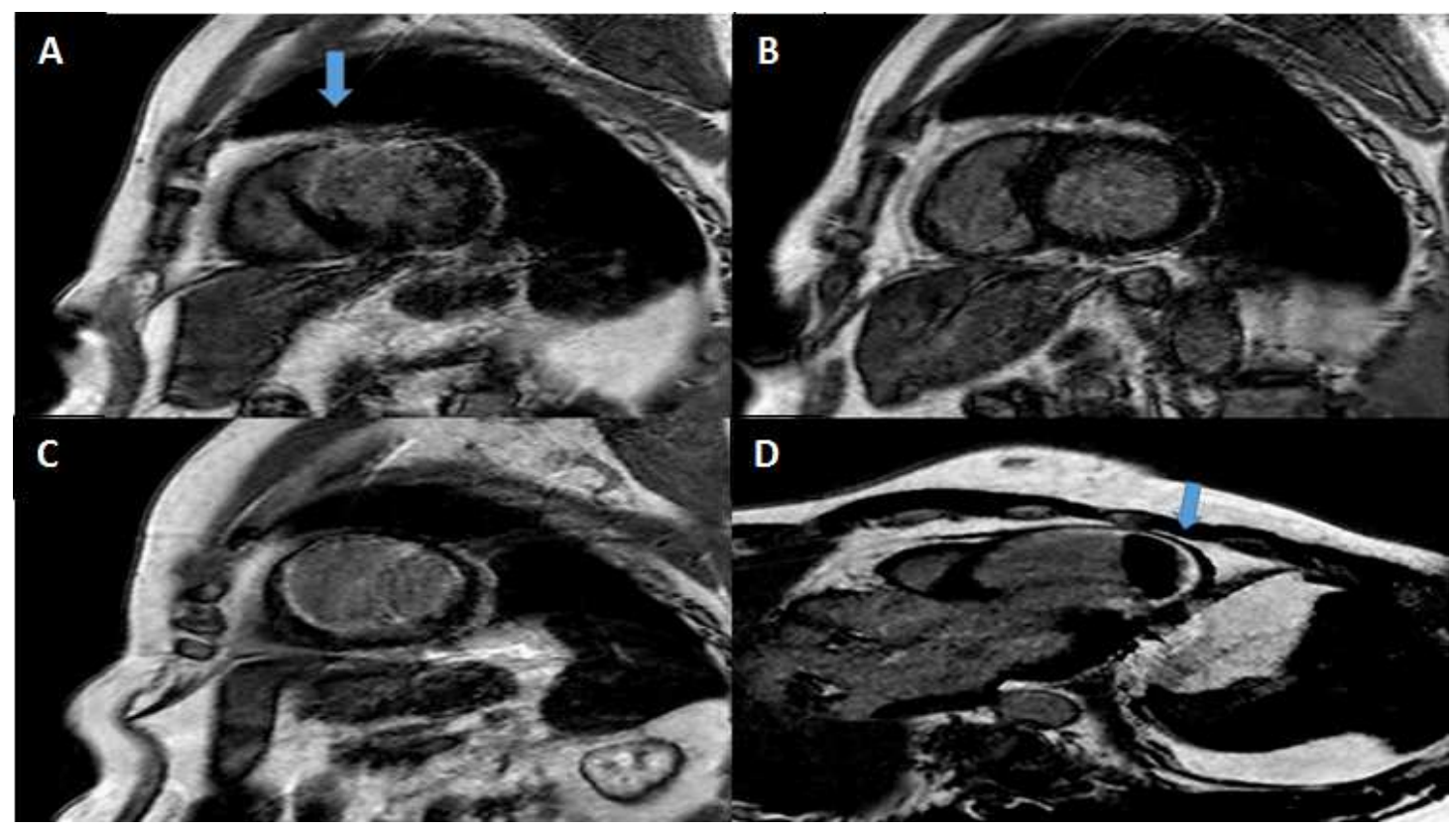

Figure 7. CMR findings. Post Gd DTPA $3 D$ IR sequence in short axis plane, three Heart chamber planes (A-D images).

MRI was of value in diagnosis of post MI complications \& other cardiac abnormalities using echocardiography as the reference standard. MRI showed a NPV of $89.4 \%$ for the evaluation of the left ventricle (LV) enlargement, for left atrial (LA) enlargement of NPV was $85.7 \%$, LV cavitary thrombus was $97.6 \%$ and as for valvular lesions, NPV was $95.2 \%$. 


\section{Discussion}

Ischemic cardiomyopathy is a result of severe CAD where ischemic etiology of cardiomyopathy is defined as the presence of any epicardial coronary vessels with significant stenosis (more than $50 \%$ ) or any history of MI or coronary revascularization (either percutaneous transluminal coronary angioplasty or coronary artery bypass grafting) [6]. Myocardial revascularization is an effective option for patients with viable myocardium where it can improve ventricular dysfunction and long-term survival, whereas revascularization in patients with predominantly nonviable myocardium exposes the patient to the unnecessary risk of invasive procedures and mortality [7]. Therefore, distinguishing dysfunctional but viable myocardium from nonviable tissue after acute or chronic ischemia has important implications for the therapeutic management of patients with CAD.

According to personal data in our study, the mean age of group A was $59.1 \pm 7.5$ with more predominance within male patients (21 male and 7 females). The mean age of group B was $53.5 \pm 11.8$ with the same predominance within male patients as group A (75\%).

As regard the clinical characteristic data, patients with diabetes mellitus were (78.6\%) followed by hypertension $(57.1 \%)$ and history of MI (39.3\%) in the group A. the reason is that important mechanisms of diabetic cardiomyopathy are attributed to metabolic disturbances, cardiac autonomic neuropathy, and insulin resistance [8]. Whilst number of the patients with diabetes mellitus were equal to ones with hypertension $(57.1 \%)$ in the group B. This may be explained that hypertension doubles the risk of cardiovascular disease and significantly accelerates the development of atherosclerosis and the prevalence of hypertension in type 2 diabetes mellitus is as high as $70-80 \%$ [9]. There are other risk factors may have role in ischemic cardiomyopathy such as Adiposity which is assessed as elevated body mass index (BMI) and smoking. In this study, we found that overweight (BMI $>25)$ and smoking in group A were $100 \%$ and $60.7 \%$ respectively while overweight (BMI>25) and smoking in group B were $75 \%$ and $57.1 \%$ respectively.

Echocardiographic data in ischemic cardiomyopathy:

According to the Echocardiographic data, the wall motion (WM) abnormality was detected in all patients (100\%) of group A and $75 \%$ of group B. the Akinetic segments were around half the patients of group A $(53.8 \%)$ while were $18.1 \%$ in group B. the hypokinetic segments were $36.8 \%$ in group $\mathrm{A}$ and $65.1 \%$ in group $\mathrm{B}$. this is due to the presence and maturity of collaterals and differences in the ischemic tolerance or metabolic characteristics of the myocardium involved [10]. In our study We found in group A that the mean LVESD was $4.3 \pm 0.9 \mathrm{~cm}$, the mean LVEDD was $5.4 \pm$ $0.7 \mathrm{~cm}$ and the mean EF was $32.3 \% \pm 12.1$, whilst in group B we found the mean LVESD was $3.6 \pm 0.8 \mathrm{~cm}$, the mean LVEDD was $4.7 \pm 0.8 \mathrm{~cm}$ and the mean EF was $40.1 \% \pm 9.9$. These results are in line with Rácz I et al; 2015 [10] that investigated 57 patients with myocardial infarction and reported that the mean of LVEF $49.9 \% \pm 5.3$, mean of
LVEDD was $51.7 \pm 4.9 \mathrm{~mm}$ as well as mean of LVESD about $34.6 \pm 4.7 \mathrm{~mm}$.

MRI in chronic ischemic cardiomyopathy (group A)

In this study, twenty-eight patients had chronic ischemic cardiomyopathy. Those patients were preparing for revascularization. So, viability assessment was crucial to determine who was a candidate and who was not. Some of them had previous interventions either by PCI (10 patients) \& CABG (7 patients). According to the CMR data, the wall motion (WM) abnormality was detected in almost all patients (96.4\%). The akinetic segments were (47.3\%) and the hypokinetic segments were $(40 \%)$. The mean ejection fraction (EF) was $30.4 \pm 15.5$ and this mean is in accordance with Krumm P et al; 2017 [11] that found the mean EF was $49 \%(<50 \%)$.

i) Myocardial viability: In this study, the myocardial segment viability of the ischemic patients revealed that patients with LGE $<50 \%$ (viable) who were eligible for revascularization procedures represented about $39.3 \%$ while patients with LGE $>50 \%$ (non-viable) who were not eligible for revascularization procedures represented about $60.7 \%$. In a study by Hillenbrand $H B$ et al; 2000 [12] it was found that when the segmental transmural extent of hyperenhancement was $<25 \%$, the majority $(87 \%)$ of segments showed improved function, whereas when the extent of hyperenhancement was $>75 \%$, functional recovery was unlikely, with intermediate degrees of hyperenhancement resulting in intermediate likelihood of recovery. Although we did not perform follow up studies after revascularization, we depend on previous work which showed that there was less likelihood of improvement in regional function with increased extent of infarction.

In a recent report of Romero $J$ et al; 2012 [13], the pooled reported diagnostic accuracy of CMR for viability assessment from 24 studies using criteria of end-diastolic wall thickness (4 studies), response to low dose dobutamine stress (9 studies), or LGE (11 studies) were examined. These authors report LGE CMR to have highest sensitivity (95\%) and NPV (90\%) for predicting improved regional wall motion, followed by end-diastolic wall thickness. Dobutamine CMR had highest specificity (91\%) and PPV (93\%). The overall weighted diagnostic accuracy was $70 \%$. Only changes in regional wall motion were reported, with no information on patient status, symptoms, ejection fraction improvement, or clinical events.

Furthermore, Camici PG et al; 2008 [14] revealed the sensitivity and the specificity and PPV and NPV of LGE CMR in evaluation of the myocardial viability were $95 \%$, $51 \%, 69 \%$ and $90 \%$ respectively. However, Schinkel AFL et al; 2007 [7] showed the sensitivity and the specificity of LGE CMR were $84 \%$ and $63 \%$.

ii) Myocardial perfusion by detection of diseased coronary artery territories:

As regard to myocardial perfusion that supplied by main coronary arteries, we found that the affected coronary artery was the left anterior descending artery (LAD) that was $78.6 \%$, followed by the right coronary artery (RCA) and left 
circumflex artery (LCX) that were $39.3 \%$ and $50 \%$ respectively. These results are in line with Krumm $P$ et al; 2017 [11] that announced $44 \%$ of all segments with recognized chronic myocardial infarction were supplied by the LAD, $25 \%$ by the LCX, and $31 \%$ by the RCA.

iii) Agreement between MRI and Conventional Coronary Angiography in detection of diseased coronary arteries in patients with chronic myocardial infarction (CMI):

Regarding the diagnostic performance of the cardiac MRI guided by coronary conventional angiogram in detection of diseased coronary arteries and the evaluation of the effect of the diseased coronary upon their territory segments of the left ventricle mainly in patients with chronic myocardial infarction (CMI). Our study results reported that there was an agreement between the two modalities with very high kappa agreement (0.814) and statistically significant Chi-square $(\mathrm{p}=0.001)$. By way of explanation, both modalities detected (LAD) affected territory only in $26 \%$ of the 28 patients while (LAD \& LCX) diseased territories seen in $11 \%$ and (LAD \& RCA) diseased territories seen in $18.5 \%$.

In the study of Kidambi A. et al; 2016 [15], the population of false - negative patients was $35(5 \%)$, all of whom had CMR graded as "probably normal". Overall, 230/752 (34\%) patients had true - positive findings. This study concluded that False - negative CMR results are uncommon, and more common in patients with lower angiographic myocardium at - risk. Plein S. et al; 2013 [17] enrolled prospectively 752 patients with suspected coronary artery disease, scheduled to undergo $\mathrm{CMR}$ and X-ray coronary angiography. 36 patients (4.7\%) with a false-negative CMR result were identified. Kidambi A. et al; 2016 [15] and Plein S. et al; 2013 [16] studies agreed with our results that exhibit high agreement between CMR and conventional coronary angiogram with true positive about $82.1 \%$ and false negative $3.7 \%$.

In the study of Bettencourt N. et al; 2013 [17], They found that CMR had $79 \%$ sensitivity, 95\% specificity, positive predictive value of $95 \%$, and negative predictive value of $78 \%$ using $\mathrm{x}$-ray invasive coronary angiography (XA) as a reference standard. While in the study of Chattranukulchai $P$. et al; 2010 [18], The sensitivity, specificity, positive predictive value, negative predictive value, and accuracy of the combined stress and rest perfusion with delayed enhancement imaging for detection of significant coronary artery stenosis were $76-96 \%, 79-96 \%, 87-93 \%, 83-96 \%$, and $85-95 \%$, in LAD, LCX, and RCA, respectively. Without delayed enhancement imaging, stress and rest perfusion produced slightly lower sensitivity (69-92\%), specificity (73$96 \%$ ), positive predictive value (79-93\%), negative predictive value (80-92\%), and accuracy (79-92\%). Therefore, Dipyridamole stress CMRI combined with delayed enhancement imaging yielded high diagnostic accuracy for the detection of coronary artery disease hence one of the limitations in our study is that we didn't use Dipyridamole stress perfusion study.

MRI in acute myocardial infarction (group B)

According to the myocardial functional imaging, we found in our study the wall motion (WM) abnormality was detected in $92.8 \%$ of patients. The Akinetic segments were $25.3 \%$ while the hypokinetic segments represent $65.9 \%$. The mean LVESV was $97.5 \pm 48.7 \mathrm{~mL}$, the mean LVEDV was $154.3 \pm$ $50.4 \mathrm{~mL}$ and the mean LVEF was $39.1 \% \pm 12.0$. These was similar to Manka $R$. et al; 2012 [19], that examined 22 patients and reported the mean LVEDV was $156.8 \pm 50.5$ $\mathrm{mL}$, the mean LVESV was $96.7 \pm 39.2 \mathrm{~mL}$ and the mean LVEF was $40.5 \% \pm 8.2$.

Other studies agreed with our results such as Sattar $H$ et al; 2008 [20], Kwong $R$ et al; 2006 [21] and Kim H et al; 2009 [22]. They mentioned that mean EF about 41\%, 47\% and $48 \%$ respectively. However, this is disagreement with Yoon Y et al; 2012 [23] and Nordenskjöld A et al; 2016 [24] that revealed the mean EF were $54 \%$ and $61 \%$ respectively. This is may be related to sensitivity variation of CMR in detection of left ventricular ejection fraction (LVEF) of this group patients with acute MI.

Franco $A$ et al; 2015 [25] concluded that the clinical use of delayed myocardial enhancement MR imaging is most performed for evaluation of a myocardium at risk. Bratis $K$ et al; 2017 [26] examined prospectively 22 consecutive patients with acute chest pain using Image-navigated 3-dimensional late gadolinium enhancement and they detected LGE in 5 patients with ischemic pattern, in 7 with non-ischemic pattern, while it was absent in 10 cases. There is quite agreement between this study and our study that detected LGE with ischemic pattern in 54\%, and LGE with nonischemic pattern in $29 \%$ and absent in $18 \%$ of our patients.

i. Myocardial viability:

Assessing viability in patients with AMI is important for determining patient prognosis and deciding whether revascularization is appropriate [27]. Bogaert et al; 2007 [28] mentioned that LGE in acute MI almost always involves the sub-endocardial layer, with increasing degrees of transmurality depending on the total occlusion time, while the sub endocardial extent of infarct depends on the localization of the occlusion (proximal versus distal) and the size of the perfusion bed dependent on the occluded coronary artery.

In this study, the myocardial segment viability of the ischemic patients via CMR revealed that patients with LGE < $50 \%$ (viable) who were eligible for revascularization procedures represented about $60 \%$ while patients with LGE $>50 \%$ (non-viable) who were not eligible for revascularization procedures represented about $40 \%$. In accordance with our results, Woo JS et al; 2015 [27] screened 30 patients with STEMI using other modality (twodimensional speckle tracking imaging) and announced that patients with viable myocardium were $70 \%(21 / 30)$ and ones with non-viable myocardium were $30 \%$ (9/30). Choi et al; 2001 [29] studied patients presenting with their first myocardial infarction with LGE-CMR performed within a week of the acute event. They found that the best predictor of global improvement at 2 to 3 months was the extent of dysfunctional myocardium without any or with less than $25 \%$ hyperenhancement.

ii. Myocardial perfusion by detection of diseased coronary artery territories: 
Based on myocardial perfusion that supplied by main coronary arteries, we found that the affected coronary artery was the left anterior descending artery (LAD) that was $53.3 \%$, followed by the right coronary artery (RCA) and left circumflex artery (LCX) that were $33.3 \%$ and $26.6 \%$ respectively. On contrary to our results, Krumm P et al; 2017 [11] showed that $30 \%$ of all segments with myocardial infarction were supplied by the LAD, $41 \%$ by the LCX, and $29 \%$ by the RCA.

iii. Risk stratification \& prognostic indices:

Regarding to risk stratification \& prognostic indices, Roes S. D et al; 2007 [30] found that the infarct size on contrastenhanced MRI being superior to LVEF and LV volumes for predicting long-term mortality in patients with myocardial infarction. In our study, we found the mean infarct size was $22.96 \pm 14.6 \%$ in line with the result of Bhatia $V$ et al; 2015 [31] that was $27.2+17.4 \%$ of LV. Stone G. W et al; 2016 [32] recommended that Acute MI size should be preferred to chronic MI size as a surrogate endpoint, because acute MI size is already prognostic, and this would reduce sample size and result in fewer dropouts.

Microvascular obstruction was defined as any hypoenhanced area present within the hyper-enhanced infarcted region on delayed contrast images. The presence and absolute amount of MVO are associated with adverse left ventricular remodeling and prognosis [33]. Bhatia $V$ et al; 2015 [31] demonstrated the presence of microvascular obstruction in $14(53.8 \%)$ of the patients. WU K et al; 1998 [34] studied 44 patients with acute MI $10+6$ days after infarction. Microvascular obstruction was present in 11 $(25 \%)$ of their patients. Our results correlated with their findings that microvascular obstruction was present in 8 patients (28.6\%), associated with larger infarct size.

iv. Additive role of T2WI sequence used in acute myocardial infarction:

For assessment of myocardial edema \& myocardium salvage index in patients with acute ischemic injury, T2weighted CMR, reliant on its sensitivity to water bound protons, permits differentiation between acute and chronic ischemic cardiomyopathy. Several studies have shown that T2-weighted imaging sensitively detects acute infarct associated edema [35]. José $V$ et al; 2009 [35] detected the presence of tissue edema in 117 (90.6\%) of 134 patients. This result is the same as our study that found in $89.3 \%$ of our patients in this group. Dall'armellina et al; 2011 [36] had established that myocardial edema is maximal and constant over the first week after myocardial infarction, providing a stable window for the retrospective evaluation of area at risk. Unfortunately, although edema imaging was performed at 5 to 7 days, LGE was not done at this time point in this study, and hence, one cannot directly compare the LGE findings of this study with the earlier studies.

Study outcomes

i. Correlation between CMR and the 2D Echo in evaluation of LV functionality of patients with acute and chronic myocardial infarction:

Although echocardiography is commonly used to evaluate cardiac function after MI, CMR may provide more accurate functional assessment but has not been adequately compared with echo. In our study, there was positive very high correlation between the two modalities in evaluation of LV Functionality indices (WMA Segment Number, LVESD, LVEDD and LVEF) with Pearson's Correlation Coefficient $(0.824,0.914,0.969$ and 0.952 respectively) and statistically significant difference in all parameters $(p<0.001)$. Regarding mean difference between CMR and Echo, Echo underestimated left ventricular (LV) diameters (by $10 \mathrm{~mm}$ for end-diastolic, $50 \mathrm{~mm}$ for end-systolic volume, both $\mathrm{p}<$ 0.005 ) but overestimated LV ejection fraction (LVEF) (by 3.5 percentage point, $\mathrm{p}=0.004)$. CMR was much more sensitive to detection of segmental wall motion abnormalities ( $\mathrm{p}<$ 0.001). Therefore, this intra subject comparison after MI found large, systematic differences between CMR and echo measures of diameters and LVEF despite high inter-modality correlations, with echo underestimating each metric except LVEF this was overestimated.

Our study did not directly determine the reasons for underestimations of diameters by echo compared with CMR. Kühl HB et al; 2004 [37] mentioned that a complete evaluation of segmental myocardial function cannot be performed in all patients owing to limitations of the ECHO imaging window with difficulties in defining endocardial contours. This is a concern in patients with advanced pulmonary disease, with high body mass index, and who have undergone thoracic surgery. However, of clinical importance, CMR may be preferred when small to moderate serial changes in these metrics are clinically important. In the study of Gardner BI; 2009 [38], 47 patients with MI were studied by both echo and CMR within 60 min of each other. This study has excellent agreement with our study that revealed measures of volume and function correlated moderately well between CMR and echo $(r=0.54$ to 0.75 , all $\mathrm{p}<0.001)$. Echo underestimated left ventricular (LV) volumes (by $69 \mathrm{ml}$ for end-diastolic, $35 \mathrm{ml}$ for end-systolic volume, both $\mathrm{p}<0.001)$ and CMR was much more sensitive to detection of segmental wall motion abnormalities $(\mathrm{p}<0.001)$ but on contrary to our result, LV ejection fraction (LVEF) was underestimated (by 4 percentage point, $\mathrm{p}=0.02$ ).

Our finding agreed with Hoffmann R et al; 2004 [39] who stated that the mean differences between EF defined by echocardiography images and EF by MRI were below $5 \%$. Whilst Dewey $M$ et al; 2006 [40] mentioned that EF comparisons of non-contrast 2D Echo with MRI were fairly accurate for assessment with limits of agreement of $21.2 \%$ and also, Pellikka PA et al; 2018 [41] mentioned that the mean absolute differences of LVEF by echocardiography and CMR were all greater than $5 \%$. This may be attributed to large number of study population and selection bias in the last two studies.

Based on a Bland-Altman plot that considered another method for measurement of agreement between CMR and 2D Echo. Among 56 patients in our study, the mean EF by MRI was $34.8 \%$ and the mean EF by echocardiography was $36.2 \%$ with a mean difference of $4.6 \%$. Furthermore, SD of bias was 
of 3.67 and $95 \%$ limits of agreement were 9.94: -7.83 . These findings agree with Hussein RS. et al; 2013 [42] that reported the mean EF by MRI was $29 \%$, the mean EF by echocardiography was $33.3 \%$ with a mean difference of $4.3 \%$, SD of bias was of 6.73 and $95 \%$ limits of agreement were $-8.90-7.5$.

ii. Agreement between ECHO and MRI in evaluation of segmental wall motion abnormality (SWMA):

We found good agreement between cardiac magnetic resonance and echocardiography regarding segmental wall motion with Cohen's Kappa is (0.76), $P$ value of (0.011). In a study performed by Kühl et al; 2004 [37], the authors found that agreement between echo and MRI regarding SWMA was moderate $(\kappa=0.47)$ with echo showing significantly lower mean scores than MR imaging. Yang et al; 1998 [43] also compared 2D-echo with gradient-echo CMR for the evaluation of SWMA in patients with good and poor echo image quality. The authors found good agreement $(\kappa=0.79)$ regarding SWMA depiction between echo and CMR imaging in the patients with good echo image quality. As regards patients with poor echo image quality, MR imaging was superior for the visualization of wall segments. On contrary to our results, Hussein $R$ et al; 2013 [42] found Poor agreement between cardiac magnetic resonance and echocardiography regarding segmental wall motion with Kappa of $0.195, P$ value of $<0.001$.

Per-segment agreement between echocardiography and MRI as regards the detection of segmental wall motion abnormality in the 476 segments in our study, it was found that there was high agreement between the two modalities in evaluation of the SWMA as both cardiac MRI and 2D Echo detected $1.8 \%$ with no motion abnormality, hypokinesia in 16 (28.6\%) patients and Akinesia in $2(3.6 \%)$ patients. our findings are close enough to Hussein $R$ et al; 2013 [42] that revealed Concordant segments between MRI and ECHO were as follows: 140 segments (22.3\%) showing hypokinesia, 39 segments (6.2\%) showing akinesia. However, the percentage of segments showing no motion abnormality by two modalities was $19.1 \%$.

iii. (C) Other values of CMR over echocardiography:

In our study, group A revealed that $57.1 \%$ of patients had post-MI complication on MRI, while in group B, patients had post-MI complication represent $32.1 \%$. In this study, we tried to evaluate other benefits of CMR over Echocardiography including diagnosis of Post MI complications using echocardiography as the reference standard. Left ventricle (LV) and Left atrial (LA) enlargement by MRI displaying NPV of $89.4 \%$ with $95 \%$ CI of $69.8-96.7 \%$ and NPV of $85.7 \%$ with $95 \%$ CI of 64.7 - 94.2\% respectively. CMR was used in detecting left ventricular cavitary thrombus by MRI using echocardiography as reference standard showed NPV of $97.6 \%$ with $95 \%$ CI of $87.9-99.4 \%$. Also, CMR was of value in diagnosis of valvular lesions using echocardiography as reference standard showed NPV of $95.2 \%$ with $95 \%$ CI of $81.8-99.5 \%$. These results are in concordant with Hussein RS. et al; 2013 [42] that showed a
NPV of $87.5 \%$ with $95 \%$ CI of $61.65-98.45 \%$ for evaluation of the left ventricle (LV) enlargement by MRI using echocardiogram as a reference standard as well as for left atrial (LA) enlargement of NPV was $80.95 \%$ with $95 \%$ CI of $58.09-94.55 \%$, LV cavitary thrombus was $96.87 \%$ with $95 \%$ CI of $83.78-99.92 \%$ and for valvular lesions was $92.59 \%$ with $95 \%$ CI of $75.71-99.09 \%$.

\section{Conclusion}

With the increasing number of patients with ischemic heart failure, information on myocardial viability \& cardiac functionality is needed to guide patient treatment. On comparing the results of CMR and echocardiography regarding global LV functional (EF), high concordance between the two modalities was noted with mean difference of $4.3 \%$.

There were certain drawbacks in our study, only patients who were stable and could withstand the cardiac MRI examination were included. The infarct size was estimated within a week of acute myocardial infarct and may represent an overestimation of the final infarct size due to edema. Final infarct size can be assessed on follow up imaging that couldn't be done due to financial constraints to the patients and difficult geographical terrain in this part of the country, thus estimation of final infarct size was not taken. Myocardial tagging in the patients could have added by estimating the myocardium at risk. We did not incorporate these as limited sequences were taken to reduce the time of acquisition of relatively sick MI patients in MR gantry. Further these sequences could have more relevant if follow up was done. The wall motion abnormalities were visually assessed and could be subjected to observer bias. One of our limitation is using LGE CMRI alone in assessment of cardiac viability without integration of other modalities such as dobutamine stress echocardiography, SPECT and PET that yield high diagnostic accuracy for evaluation of myocardial viability.

In conclusion, CMR was a feasible and quantitative method of assessing myocardial viability. Delayed contrast enhanced cardiac MRI examination can give both morphological and functional information which have important therapeutic as well as prognostic implications.

\section{Conflicts of Interest}

There are no conflicts of interest.

\section{Acknowledgements}

The authors would like to thank the Assiut University Radiology Department for helping to perform this work.

\section{References}

[1] Fox K, Steg P, Eagle K, Goodman S, Anderson F, Granger C, et al. GRACE investigators. Decline in rates of death and heart failure in acute coronary syndromes, 1999-2006. JAMA. 2007; 2 (297): 1892-900. 
[2] Ganame J, Messalli G, Dymarkowski S, Rademakers F, Desmet W, Van de Werf F, et al. Impact of myocardial haemorrhage on left ventricular function and remodelling in patients with reperfused acute myocardial infarction. European heart journal. 2009; 3 (30): 1440-9.

[3] Dall'Armellina E, Karamitsos T, Neubauer S ,Choudhury R. $\mathrm{CMR}$ for characterization of the myocardium in acute coronary syndromes. Nature Reviews Cardiology. 2010; 7 (11): 624-34.

[4] Lloyd-Jones D, Adams R, Brown T, Carnethon M, Dai S, De Simone G, et al. Writing Group Members. Executive summary: heart disease and stroke statistics-2010 update: a report from the American Heart Association. Circulation. 2010; 121 (7): 948-54

[5] Grothues F, Moon J, Bellenger N, Smith G, Klein H, Pennell D. Interstudy reproducibility of right ventricular volumes, function ,and mass with cardiovascular magnetic resonance. American heart journal. 2004; 147 (2): 18-23.

[6] Felker G, Shaw L, O'Connor C. Standardized Definition of Ischemic Cardiomyopathy for Use in Clinical Research. JACC. 2002; 39 (1): 210-8.

[7] Schinkel A, Bax J, Poldermans D. Clinical assessment of myocardial hibernation. Heart J 2005; 91 (1): 111-7.

[8] Hueb T, Rocha M, Siqueira S. Impact of diabetes mellitus on ischemic cardiomyopathy. Five-year follow-up. REVISIONDM trial. Diabetol Metab Syndr. 2018; 10 (1): 19.

[9] Špinar J. Hypertension and ischemic heart disease,. Cor et Vasa. 2012; 54 (6): 433-8.

[10] Rácz I, Fülöp L, Kolozsvári R. Wall motion changes in myocardial infarction in relation to the time elapsed from symptoms until revascularization. Anatol J Cardiol. 2015; 15 (5): $363-70$

[11] Krumm P. Cardiac MRI left ventricular global function index and quantitative late gadolinium enhancement in unrecognized myocardial infarction. European journal of radiology. 2017; 92 (1): 11-6.

[12] Hillenbrand H, Kim R, Parker M. Early assessment of myocardial salvage by contrast-enhanced magnetic resonance imaging. Circulation 2000; 102 (1): 1678-83.

[13] Romero J, Xue X, Gonzalez W, Garcia M. CMR imaging assessing viability in patients with chronic ventricular dysfunction due to coronary artery disease: a meta-analysis of prospective trials. JACC: Cardiovascular Imaging. 2012; 5 (5): 494-508.

[14] Camici P, Prasad S, Rimoldi O. Stunning, hibernation, and assessment of myocardial viability. Circulation. 2008; 117 (1): 103-14.

[15] Kidambi A, Sourbron S, Maredia N. Factors associated with false-negative cardiovascular magnetic resonance perfusion studies: A Clinical evaluation of magnetic resonance imaging in coronary artery disease (CE-MARC) substudy. J Magn Reson Imaging. 2016; 43 (3): 566-73.

[16] Plein S, Kidambi A, Sourbron S, Maredia N, Uddin A, Motwani $M$, et al. Associated factors for a false negative cardiovascular magnetic resonance perfusion study: a CEMARC substudy. Journal of Cardiovascular Magnetic Resonance. 2013; 15 (1): P214.
[17] Bettencourt N, Ferreira N, Chiribiri A, Schuster A, Sampaio F, Santos L, et al. Additive value of magnetic resonance coronary angiography in a comprehensive cardiac magnetic resonance stress-rest protocol for detection of functionally significant coronary artery disease: a pilot study. Circulation. 2013; 6 (5): 730-8.

[18] Chattranukulchai P, Tumkosit M, Cholteesupachai J, Chaipromprasit J. Diagnostic accuracy of combined dipyridamole stress perfusion and delayed enhancement cardiovascular magnetic resonance imaging for detection of coronary artery disease. Asian Biomedicine. 2010; 4 (1): 1925 .

[19] Manka R, Kozerke S, Rutz A, Stoeck C, Boesiger P, Schwitter J. A CMR study of the effects of tissue edema and necrosis on left ventricular dyssynchrony in acute myocardial infarction: implications for cardiac resynchronization therapy. Journal of Cardiovascular Magnetic Resonance. 2012; 14 (1): 47.

[20] Sattar H, Kwong R, Wu H, Vorobiof G, Gandla V, Steel K, et al. CLINICAL PERSPECTIVE. Circulation. 2008; 118 (10): 1011-20.

[21] Kwong R, Chan A, Brown K, Chan C, Reynolds H, Tsang S, et al. CLINICAL PERSPECTIVE. Circulation. 2006; 13 (23): 2733-43.

[22] Kim H, Farzaneh-Far A, Kim R .Cardiovascular magnetic resonance in patients with myocardial infarction: current and emerging applications. Journal of the American College of Cardiology. 2009; 55 (1): 1-6.

[23] Yoon Y, Kitagawa K, Kato S, Ishida M, Nakajima H, Kurita T, et al. Prognostic value of coronary magnetic resonance angiography for prediction of cardiac events in patients with suspected coronary artery disease. Journal of the American College of Cardiology. 2012; 60 (22): 2316-22.

[24] Nordenskjöld A, Hammar P, Ahlström H, Bjerner T, Duvernoy $\mathrm{O}$, Eggers $\mathrm{K}$, et al. Unrecognized Myocardial Infarction Assessed by Cardiac Magnetic Resonance Imaging-Prognostic Implications. PloS one. 2016; 17 (2): $\mathrm{e} 0148803$.

[25] Franco A, Javidi S, Ruehm S. Delayed Myocardial Enhancement in Cardiac Magnetic Resonance Imaging. J Radiol Case Rep. 2015; 9 (6): 6-18.

[26] Bratis K, Henningsson M, Grigoratos C, Dell'Omodarme M, Chasapides K, Botnar R, et al. Image-navigated 3-dimensional late gadolinium enhancement cardiovascular magnetic resonance imaging: feasibility and initial clinical results. J Cardiovasc Magn Reson. 2017; 19 (1): 97.

[27] Woo J, Yu T, Kim W, Kim K, Kim W. Early prediction of myocardial viability after acute myocardial infarction by twodimensional speckle tracking imaging. J Geriatr Cardiol. 2015; 12 (5): 474-81.

[28] Bogaert J, Kalantzi M, Rademakers F. Determinants and impact of microvascular obstruction in successfully reperfused ST-segment elevation myocardial infarction. Assessment by magnetic resonance imaging. Eur Radiol. 2007; 17 (1): 257280 .

[29] Choi K, Kim R, Gubernikoff G. Transmural extent of acute myocardial infarction predicts long-term improvement in contractile function. Circulation. 2001; 104 (1): 1101-7. 
[30] Roes S, Kelle S, Kaandorp T. Comparison of myocardial infarct size assessed with contrast- enhanced magnetic resonance imaging and left ventricular function and volumes to predict mortality in patients with healed myocardial infarction. Am J Cardiol. 2007; 100 (1): 930-6.

[31] Bhatia V, Sood R, Dhiman D. Predictors of acute myocardial infarct size in STEMI patients receiving thrombolytic therapy: A delayed contrast enhanced cardiac MRI study. Indian Heart J. 2015; 67 (2): 122-7.

[32] Stone G, Selker H, Thiele H. Relationship between infarct size and outcomes following primary PCI: patient-level analysis from 10 randomized trials. J Am Coll Cardiol. 2016; 67 (1): 1674-83.

[33] Vermes E, Carbonea I, Friedricha M. Patterns of myocardial late enhancement: Typical and atypical features. Archives of Cardiovascular Disease 2012; 105 (1): 300-8.

[34] Wu K, Zerhouni E, Judd R. Prognostic significance of microvascular obstruction by magnetic resonance imaging in patients with acute myocardial infarction. Circulation. 1998; 97 (1): 765-72.

[35] Grover S, Srinivasana G, Selvanayagama J. Evaluation of Myocardial Viability With Cardiac Magnetic Resonance Imaging. Prog Cardiovasc Dis. 2011; 54 (1): 204-14.

[36] Dall'armellina E, Karia N, Lindsay A. Dynamic changes of edema and late gadolinium enhancement after acute myocardial infarction and their relationship to functional recovery and salvage index. Circ Cardiovasc Imaging 2011; 4 (1): 228-36.

[37] Kühl H, Spuentrup E, Wall A, Franke A, Schröder J, Heussen $\mathrm{N}$, et al. Assessment of myocardial function with interactive non-breath-hold real-time MR imaging: comparison with echocardiography and breath-hold cine MR imaging. Radiology. 2004; 231 (1): 198-207.
[38] Gardner B, Bingham S, Allen M. Cardiac magnetic resonance versus transthoracic echocardiography for the assessment of cardiac volumes and regional function after myocardial infarction: an intrasubject comparison using simultaneous intrasubject recordings. Cardiovascular Ultrasound. 2009; 7 (1): 38 .

[39] Hoffmann R, von Bardeleben S, ten Cate F, Borges A, Kasprzak J, Firschke C, et al. Assessment of systolic left ventricular function: a multi-centre comparison of cineventriculography, cardiac magnetic resonance imaging, unenhanced and contrast-enhanced echocardiography. European heart journal. 2004; 26 (6): 607-16.

[40] Dewey M, Müller M, Eddicks S, Schnapauff D, Teige F, Rutsch W, et al. Evaluation of global and regional left ventricular function with 16-slice computed tomography, biplane cineventriculography, and two-dimensional transthoracic echocardiography: comparison with magnetic resonance imaging. Journal of the American College of Cardiology. 2006; 48 (10): 2034-44.

[41] Pellikka P, She L, Holly T, Lin G, Varadarajan P, Pai R, et al. Variability in ejection fraction measured by echocardiography, gated single-photon emission computed tomography, and cardiac magnetic resonance in patients with coronary artery disease and left ventricular dysfunction. JAMA. 2018; 1 (4): e181456.

[42] Hussein R, Ibrahim A, El-Hameed A, El-Fiky A, Tantawy W. Does CMR have an additive role over echo in evaluating ischemic LV dysfunction? The Egyptian Journal of Radiology and Nuclear Medicine. 2013; 44 (3): 475-82.

[43] Yang P, Kerr A, Liu A, Liang D, Hardy C, Meyer C, et al. New real-time interactive cardiac magnetic resonance imaging system complements echocardiography. Journal of the American College of Cardiology. 1998; 32 (7): 2049-56. 